CONCURSO ESTUDIANTIL DE DISEÑO

\title{
Siempre contigo: Una ayuda en el tratamiento de la depresión en adultos mayores
}

\author{
Andrés E. Acevedo H., Gabriel García G., Ivan \\ Martínez G., Cosijoeza Melchor N.
}

Publicado: 21 Septiembre 2016

\begin{abstract}
Resumen
Con el aumento de la perspectiva de vida de los mexicanos, viene un cambio importante en el paradigma y la conformación de la población en México. Con ésto, el incremento de enfermedades crónicas, como la diabetes y la hipertensión, se han convertido en un grave problema de salud pública. En cambio, la depresión, presente en la gran mayoría de la población de adultos mayores, pasa mayormente desapercibida. Siempre Contigo es una aplicación móvil en una tableta electrónica desarrollada con la forma de un portarretratos tradicional que provee un canal de comunicación entre adultos mayores con depresión leve a moderada con estudiantes interesados en el conocimiento y experiencia atesorada por adultos mayores. De esta manera, Siempre Contigo se constituye en un auxiliar en el tratamiento de la depresión en adultos mayores enmarcado dentro de un tratamiento de psicoterapia.
\end{abstract}

Palabras clave: Depresión; Psicoterapia; Adultos mayores; UCD; Tableta.

\section{Introducción}

El desarrollo social del país conlleva un sinnúmero de efectos tanto positivos como negativos en el bienestar de la sociedad. Uno de ellos, es el crecimiento en la edad promedio en México. El número de adultos mayores se duplicó en las últimas décadas, pasando de 5 a 11.7 millones de 1990 a 2014.

Una visión integral invita a reflexionar sobre los desafíos de la población que transita o transitará por esta etapa de vida. De acuerdo a las proyecciones de población que estima la CONAPO, para 2025 y 2050 el monto de adultos mayores aumentará a 17.2 y 32.4 millones, respectivamente [1] (ver Figura 1).

Muchos de los fallecimientos en este grupo de población se producen por enfermedades crónico degenerativas, entre las que destacan la diabetes mellitus (16.9\%), las enfermedades isquémicas del corazón (16.5\%), las enfermedades cerebrovasculares $(7 \%)$, las enfermedades crónicas de las vías respiratorias inferiores $(5.9 \%)$, las enfermedades del hígado

Acevedo, AE., García, G., Martínez. I., Melchor, C.

Universidad Tecnológica de la Mixteca

Km 2.5 Carretera a Acatlima

Huajuapan de León, Oaxaca

Email: andresah492@gmail.com,ggg951016@gmail.com,

ivanmtz49@hotmail.com,cosi_deep@hotmail.com
(4.5\%) y las enfermedades hipertensivas $(4.3 \%)$ [2]

De acuerdo con la Organización Mundial de la Salud (OMS), en México, más del $20 \%$ de la población de 60 años y más sufre algún trastorno mental o neural, siendo la demencia y la depresión los padecimientos neuropsiquiátricos más frecuentes. Estimaciones de la OMS señalan que la depresión es la enfermedad mental que más afecta a la población y que para el año 2020 será el segundo padecimiento de mayor carga de morbilidad en el mundo [3].

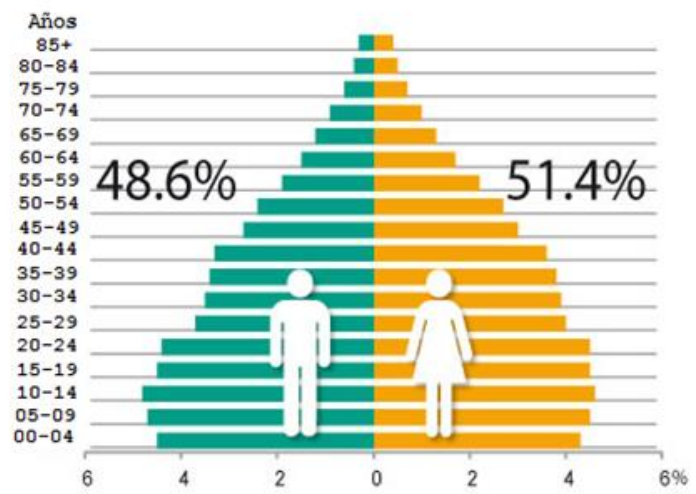

Figura 1. Crecimiento del número de los adultos mayores en la población de México [1]

De acuerdo con la OMS, existen tratamientos psicológicos para los diferentes tipos de depresión leve y moderada que consiste en terapias cognitivas conductual y psicoterapias interpersonales. Entre estos tratamientos, la psicoterapia tiene como fin ayudar al paciente a conocerse mejor y cambiar sus malas formas de pensar, sentir y actuar [4].

Por esto, nuestra pregunta de investigación se define como: ¿Podrá la tecnología ser un auxiliar útil en el tratamiento de psicoterapia para la depresión en adultos mayores?

Desafortunadamente, los casos de depresión en adultos mayores en nuestro país pasan mayormente inadvertidos, por lo que no existen cifras oficiales que nos den una idea de la magnitud del problema en nuestro estado de Oaxaca [5].

Trabajos similares [6, 7, 8, 9] han buscado aliviar los síntomas de la depresión en adultos mayores. Ninguno de éstos ha sido desarrollado en un contexto mexicano, ni tampoco en ambientes de pocos recursos por lo que un gran número de preguntas queda por resolver. 


\section{Objetivo del proyecto}

Los síntomas de la depresión pueden ser tratados. Existen diversas formas de ayuda, una de las más populares es conocida como la psicoterapia. Por medio de la psicoterapia, los psicólogos ayudan a las personas a llevar adelante vidas más felices, saludables y productivas. Como parte de un proceso continuo, realizamos algunas evaluaciones iniciales. Para nuestro proyecto, utilizamos la Evaluación de Depresión Geriátrico del Instituto Nacional de las Personas Adultas Mayores (INAPAM). Esta evaluación inicial confirmó que, de los tres adultos mayores evaluados para nuestro estudio, dos de ellos mostraron tener un nivel de depresión de leve a moderado [11].

Uno de los síntomas típicos de la depresión es el sentimiento de inutilidad o de culpa excesivos o inapropiados [4] que siente el paciente. Superar esto es uno de los grandes retos de la psicoterapia. Siempre Contigo es un dispositivo que, a través de video chats y la reafirmación de mensajes positivos (con música, fotografías de familiares, mensajes motivacionales y nutrimentales) ayude a los adultos mayores con depresión leve y moderada para que puedan tener una mejor calidad de vida.

De esta forma, el video chat ayudará a los adultos mayores a sentirse útiles ante la sociedad, utilizando la experiencia que han adquirido mediante los años laborados en diversos temas, interactuando con estudiantes de diferentes niveles académicos o interesados en su área del conocimiento que los adultos mayores posean.

\section{Metodología utilizada}

Con el objetivo de garantizar la usabilidad y mejorar la Experiencia de Usuario (UX), se decidió utilizar la metodología del Diseño Centrado en el Usuario (UCD por sus siglas en Inglés), considerado especialmente a los usuarios adultos mayores. La metodología UCD nos permite, a partir de un proceso iterativo, mejorar la facilidad de uso de nuestros desarrollos. La Figura 2, abajo, nos muestra este proceso:

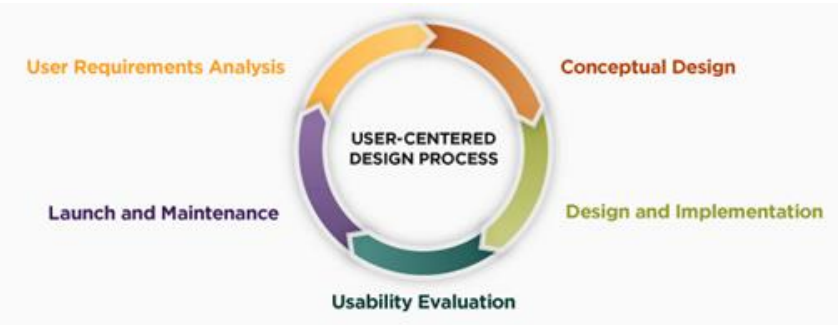

Figura 2. Diagrama de UCD (Michigan State University) [10]

\subsection{Etapas desarrolladas}

\subsubsection{Análisis de requerimientos.}

Realizamos una extensiva investigación documental sobre el tema de la depresión y su tratamiento a través de la psicoterapia. Además, realizamos consultas con la Psic. María de la Luz Palacios, del Instituto de Diseño de nuestra universidad. De esta manera definimos claramente el problema y planteamos posibles soluciones.

Enmarcamos nuestro desarrollo dentro de la terapia de psicoterapia en el aspecto de hacer sentir mejor a los adultos mayores a través de mensajes que reafirmen una actitud positiva.

El desarrollo de cinco Estudios Contextuales con adultos mayores, sus familiares que los cuidan y estudiantes en psicología auxiliares en la terapia de pacientes en asilos, nos permitieron entender las necesidades de cada grupo de usuarios y su contexto.

\subsubsection{Diseño conceptual}

A partir de esta información se desarrollaron tres Personas para los diferentes usuarios de Siempre Contigo (Adultos Mayores, sus Familiares que los cuidan y Estudiantes interesados en el conocimiento y experiencia de los ancianos).

Durante el Estudio Contextual nos dimos cuenta que los adultos mayores contaban con un gran número de fotografías enmarcadas en sus hogares, por lo que decidimos que la forma de nuestro dispositivo fuera un marco de fotografías. La Figura 3 nos muestra uno de esos estudios.

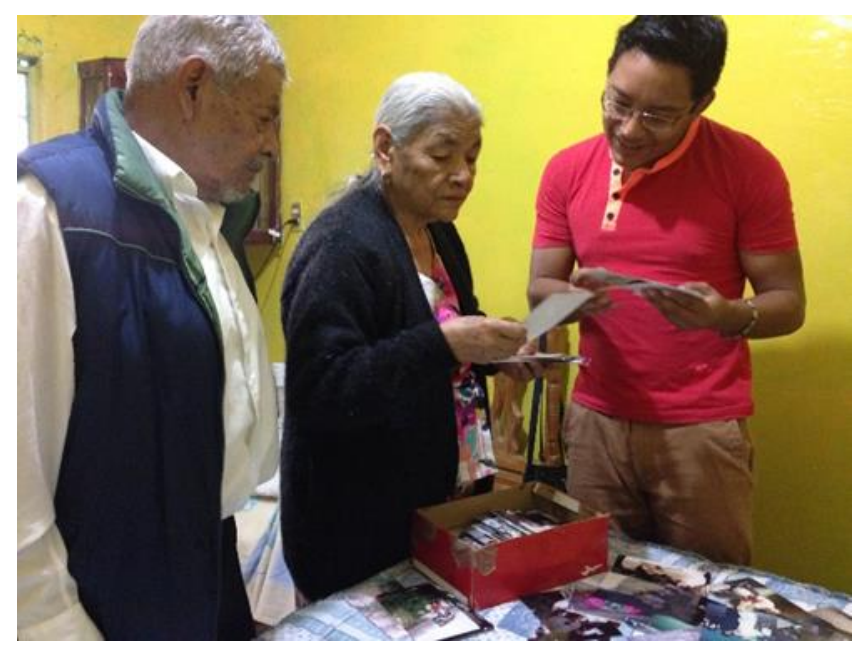

Figura 3. Estudio Contextual a la primera pareja de adultos mayores en Huajuapan de León, Oaxaca México

Inicialmente planeamos que Siempre Contigo sería un dispositivo el cual contaría con un video chat, fotos, música y mensajes motivacionales grabados por los familiares cercanos de las personas mayores con depresión. Sin embargo, nos dimos cuenta que en algunos casos, la depresión que sufren las personas mayores se debe a que sus familiares los abandonan, por lo tanto, escuchar mensajes grabados por ellos sería contraproducente. En estos casos, se generarán mensajes y se usarán fotografías genéricas para sus dispositivos. También, los usuarios nos pidieron que los juegos que se habían planeado fueran sustituidos por libros, ya que ellos preferían leer a estar jugando. Finalmente, decidimos quitar los libros debido a que nuestro dispositivo fue rediseñado a compartir la experiencia de los adultos mayores y reforzar el sentimiento de utilidad de los pacientes.

\subsubsection{Diseño e implementación.}

Se desarrollaron entonces una aplicación móvil para una tableta Android y una página web.

A. Aplicación móvil siempre contigo: Cuenta con dos partes, una aplicación para el adulto mayor que le permitirá hacer y recibir tanto video chats aleatorios como dirigidos con otros usuarios y estudiantes interesados en su experiencia. Además, el marco desplegará de manera aleatoria fotos de familiares y amigos, su música favorita y mensajes motivacionales. La Figura 4 muestra la interface de video chat con un estudiante. La segunda parte está dirigida a los familiares que cuidan de ellos, que les permitirán configurar el dispositivo y cargar el material multimedia para el adulto.

B. Servicio web siempre contigo: Este servicio web permitirá a los estudiantes interesados en obtener información de la experiencia y conocimiento de los adultos mayores a poder 
hacer una búsqueda y poder entonces conversar con ellos. La Figura 5 nos muestra el resultado de una búsqueda.

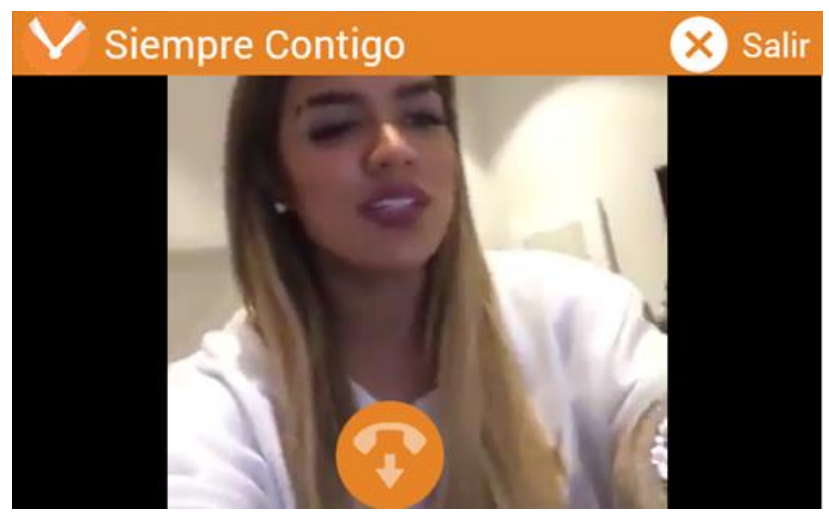

Figura 4. Este video chat fue realizado por el interés del estudiante en la experiencia del adulto mayor con depresión

\section{Siempre Contigo}

plantas $\quad a$
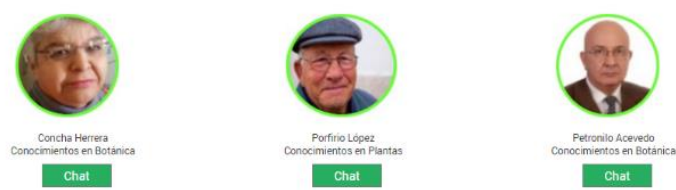

Figura 5. Esta interface muestra a los adultos mayores conectados en línea a los cuales los estudiantes pueden contactar

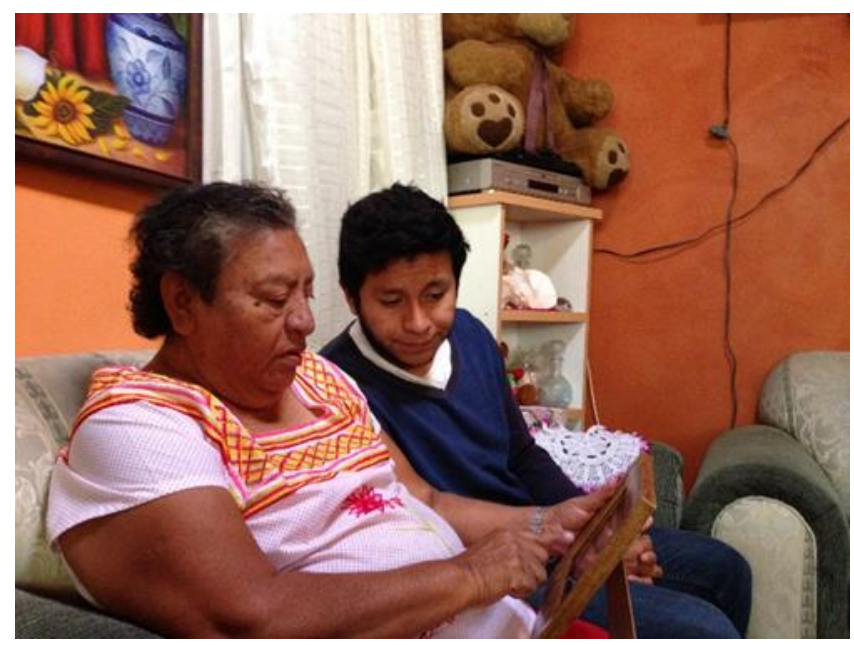

Figura 6. Evaluación de Mago de $\mathrm{Oz}$ al prototipo de alta fidelidad de la aplicación móvil de Siempre Contigo

\subsubsection{Evaluación de usabilidad}

Con el objetivo de probar nuestros prototipos con usuarios reales, se realizaron dos series de pruebas con adultos mayores y dos más con familiares que los cuidan, utilizando la técnica de Mago de Oz. Se montó una tableta Android Kingdata de 7" a un marco de madera que simula un porta-retratos tradicional de fotografías. Además se utilizó una laptop HP con un navegador Microsoft Edge con dos estudiantes probando la página web.
Nuestro objetivo fue probar el video chat para las personas mayores, además de mostrarles los mensajes y las fotografías que aparecerían en el marco. Para sus familiares, nuestro objetivo fue que conocieran el proceso de configuración de Siempre Contigo y que nos dijeran su opinión. Para los estudiantes, probamos que el proceso de darse de alta, la búsqueda y el chat en la página web fueran entendidos y que fueran útiles para ellos. La Figura 6, muestra una de estas pruebas.

\subsubsection{Resultados y rediseño}

Finalmente, en base a la retroalimentación obtenida, se planteó el rediseño del dispositivo y de las actividades realizadas con el objetivo de mejorar el proyecto de Siempre Contigo. A continuación se detallan dichos resultados y su significado.

\section{Resultados obtenidos}

Las medidas evaluadas para este tipo de pruebas se dividen en dos:

- Resultados Cuantitativos: Son los resultados obtenidos a través de mediciones cuantificables. Para nuestras evaluaciones medimos la Eficacia, entendida como el porcentaje de las tareas exitosas realizadas por el usuario y la Eficiencia, tiempo en el que se realizaron las tareas.

- Resultados Cualitativos: Son las evaluaciones de satisfacción general expresadas por el usuario de manera numérica y de manera verbal que nos indican lo que les gustó y que necesitamos cambiar del proyecto.

\subsection{Resultados de las evaluaciones}

Los resultados obtenidos de las evaluaciones a los prototipos nos proporcionan información valiosa para nuestro proyecto. Las siguientes tablas resumen estos resultados:

Tabla 1. Resultados de la evaluación con adultos mayores

\begin{tabular}{|l|l|}
\hline \multicolumn{2}{|l|}{ Evaluación Adultos Mayores } \\
\hline Eficacia & $93 \%^{*}$ \\
\hline Eficiencia & $3: 05 \mathrm{Max}$ \\
& $2: 09 \mathrm{Min}$ \\
& $2: 57 \mathrm{Prom}$ \\
\hline Satisfacción & 9.0 \\
\hline
\end{tabular}

Tabla 2. Resultados de la evaluación con familiares

\begin{tabular}{|l|l|}
\hline \multicolumn{2}{|l|}{ Evaluación Familiares } \\
\hline Eficacia & $93 \%$ \\
\hline Eficiencia & $3.23 \mathrm{Max}$ \\
& $2.43 \mathrm{Min}$ \\
& $2.83 \mathrm{Prom}$ \\
\hline Satisfacción & 8.5 \\
\hline
\end{tabular}

Tabla 3. Resultados de la evaluación con estudiantes

\begin{tabular}{|l|l|}
\hline \multicolumn{2}{|l|}{ Evaluación Estudiantes } \\
\hline Eficacia & $100 \%$ \\
\hline Eficiencia & $3.38 \mathrm{Max}$ \\
& $3.17 \mathrm{Min}$ \\
& $3.27 \mathrm{Prom}$ \\
\hline Satisfacción & 9.0 \\
\hline
\end{tabular}




\subsection{Interpretación de resultados}

La evaluación de la aplicación Siempre Contigo por las personas mayores obtuvo una evaluación alta hasta el momento en que usaron el chat. Indicaron que les da mucho gusto poder platicar con alguien que no esté en el mismo cuarto con ellos.

Tanto la aplicación de configuración como la página web recibieron muy buenas calificaciones además de que la eficacia fue muy alta. Los usuarios indicaron que las aplicaciones eran intuitivas, aunque indicaron que la presentación de la información era muy formal. Ambos grupos de usuarios indicaron que sí usarían la aplicación.

\section{Discusión}

Siempre Contigo es nuestra propuesta para auxiliar en el tratamiento psicoterapéutico de la depresión leve y moderada de adultos mayores en Estado. Está compuesta de una aplicación móvil que funciona en una tableta dentro de un marco fotográfico y una página de web donde los estudiantes interesados en aprender de estos adultos puedan video chatear con ellos, de esta manera incrementando el sentimiento de utilidad en pacientes con depresión. A través de las evaluaciones obtuvimos una serie de mejoras que resumimos aquí:

1. Los usuarios quisieran poder modificar el esquema de colores de la aplicación de acuerdo al sexo del usuario.

2. Los usuarios quisieran que las fotografías mostradas coincidan de alguna manera con la canción interpretada en ese momento.

3. Un usuario solicitó que la tableta fuera desmontable del marco, ya que lo quisiera utilizar cuando maneja, haciéndola así más práctico.

4. Los estudiantes solicitaron más información sobre el servicio y los temas de interés mostrados en la página web.

¿Es Siempre Contigo útil como ayuda en el tratamiento de la depresión? No es posible decirlo con seguridad en estos momentos. Sería necesario su utilización constante por parte de los adultos mayores y poder así evaluar su progreso. Estadísticas muestran que la psicoterapia es efectiva en el $75 \%$ de los casos [11]. Como se puede observar, las evaluaciones de usabilidad muestran una gran aceptación por parte de todos sus usuarios. Además, la Dra Luz Palacios expresó que ésta sería una aplicación muy útil para la terapia de sus pacientes con depresión. Como nos damos cuenta, es necesario implementar los cambios recomendados en una nueva iteración del proyecto, así como una nueva serie de pruebas.

\section{Agradecimientos}

Queremos agradecer a todos nuestros usuarios participantes en los Estudios Contextuales, entrevistas, pruebas y evaluaciones; especialmente a los adultos mayores, así como a sus familiares, estudiantes y doctores en psicología. Agradecemos a nuestra universidad y al UsaLab de la UTM por las facilidades prestadas.

\section{Referencias}

[1] INEGI (1990) XI Censo General de Población y Vivienda, 1990. CONAPO. Proyecciones de la Población de México, 2010-2050.

[2] INEGI (2012). Estadísticas de mortalidad, 2012. Base de datos.

[3] SSA (2013). Egresos Hospitalarios 2012. Base de datos, procesó INEGI.

[4] Depresión tratamientos, síntomas e información en DMedicina. (2009). Recuperado 01 Julio, 2016, desde http://www.dmedicina.com/enfermedades/psiquiatricas/depre sion.html

[5] Leer previene enfermedades neurodegenerativas. (2016). Recuperado 01 Julio, 2016, desde http://www.salud.oaxaca.gob.mx/leer-previeneenfermedades-neurodegenerativas/

[6] Backer, R., Sellen, K., Crosskey, S. Boscart, V., BarbosaNeves, B. (2014). Technology to reduce social isolation and loneliness. En Proceedings of the 16th international ACM SIGACCESS conference on Computers \& accessibility. Recuperado 03, Julio 2016, desde http://dl.acm.org/citation.cfm?id=2661375

[7] Machesney, D., Wexler, S., Chen, T., F. Coppola, J. Bridging the Gap for the Forgetten Population. (2014). En 2014 IEEE Long Island Systems, Applications and Technology Conference (LISAT). Recuperado desde https://www.researchgate.net/publication/271425117_Geront echnology_Companion_Virutal_pets_for_dementia_

[8] Vinicio Ferruzca, M. Process of Designing a System for Early Detection and Treatment of the State of Depression in Older Adults. (2014). Med-e-Tel 2014 Electronic Proceedings: The International eHealth, Telemedicine and Health ICT Forum for Educational, Networking and Business, At Luxemburgo. Recuperado desde https://www.researchgate.net/publication/275346851_Proces s_of_Designing_a_System_for_Early_Detection_and_Treat ment_of_the_State_of_Depression_in_Older_Adults

[9] CNA. (2014). CNA Speaking Exchange. Recuperado 25 Junio, 2016, desde https://www.youtube.com/watch?v=-S5 EfwpFOk

[10] Usability/Accessibility Research and Consulting - Michigan State University. (n.d.). Recuperado 05 Julio, 2016, desde http://usability.msu.edu/about/philosophy

[11] American Psychological Association, Entendiendo la Psicoterapia (2016). Recuperado 29 Julio, 2016, desde http://www.apa.org/centrodeapoyo/entendiendo-lapsicoterapia.aspx 